\title{
Diseño y análisis de arreglo lineal de antenas dipolo
}

\author{
Design and analysis of linear dipole antenna \\ arrangement
}

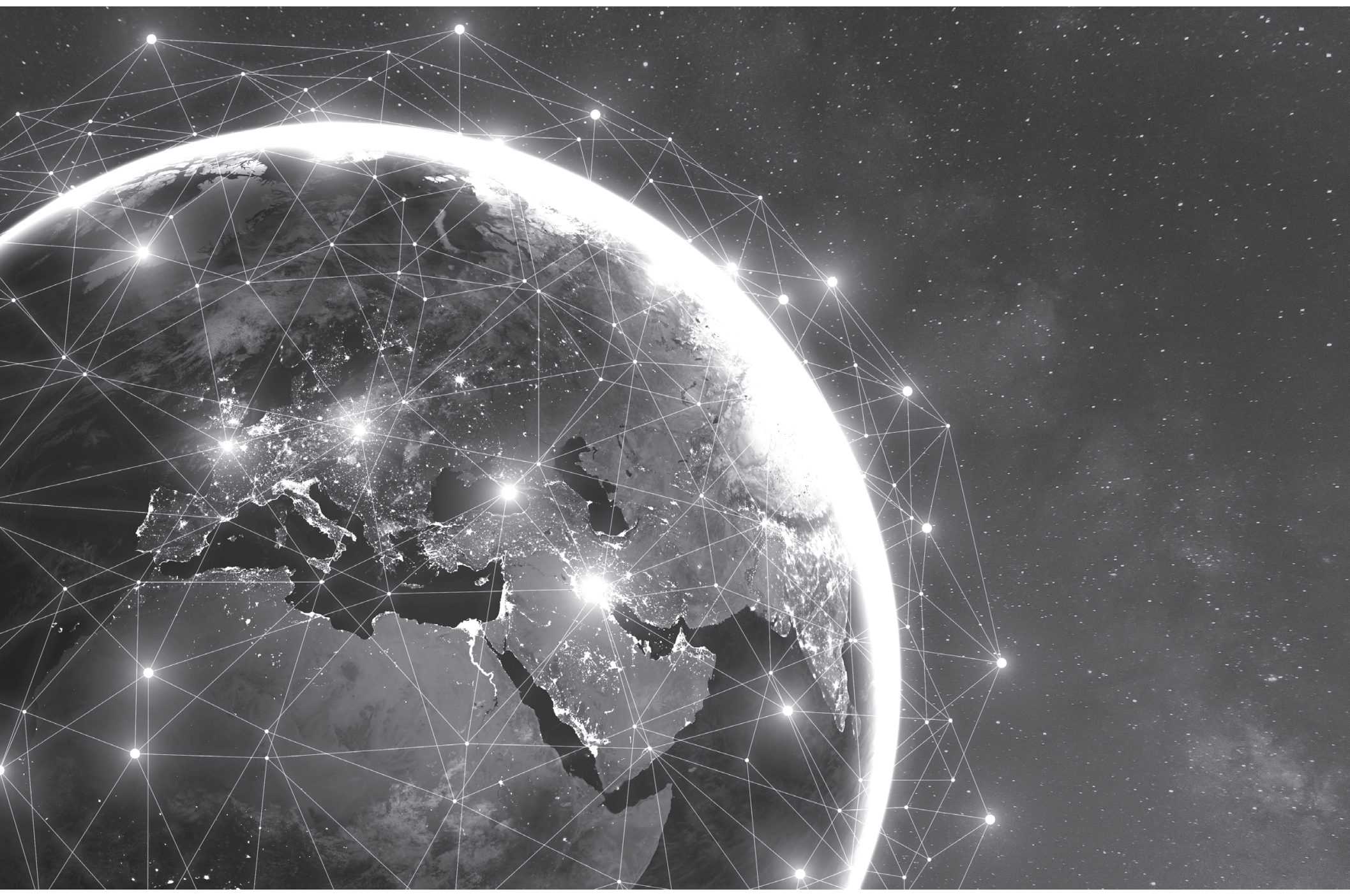




\title{
Diseño y análisis de arreglo lineal de antenas dipolo ${ }^{1}$
}

\author{
Design and analysis of linear \\ dipole antenna arrangement
}

\section{Ana Carolina Ramírez Cleves ${ }^{2}$, Edgar Javier Mantilla Bautista ${ }^{3}$}

2,3Universidad Distrital Francisco José de Caldas, Bogotá, Colombia

Artículo recibido en enero de 2017; artículo aceptado en mayo de 2017

Citación del artículo: Ramírez, A. \& Mantilla, E. (2017). Diseño y análisis de arreglo lineal de antenas dipolo.

I+D Revista de Investigaciones, 10(2) julio - diciembre de 2017, pp. 38 - 56

\begin{abstract}
Resumen
Este articulo explora el método para realizar el diseño de un arreglo de antenas; el objetivo es verificar y modelar el patrón de radiación variando características como distancia y fase. Durante el proceso se analizan diferentes posibilidades de desarrollo que cumplan con la característica de ser un sistema funcional, de fácil acceso al público y sencillo de comprender. Como resultado, se obtuvo que el sistema que cumple con el objetivo inicial y las características anteriormente indicadas es un arreglo de antenas lineal uniforme con elementos dipolo lambda medios, para cuyo desarrollo se analizan las impedancias y el desfase de los elementos comprometidos en la carta de Smith, concluyendo que este sistema es práctico y de fácil comprensión comparado con métodos como la matriz de Butler. La estructura se fabrica con elementos utilizados a diario, cumpliendo con los requerimientos inicialmente planteados.
\end{abstract}

Palabras clave: espectrómetro, antena, impedancia, sumador, dipolo (tesauro Ecured).

\begin{abstract}
This article explores the method for realizing the design of an array of antennas, the objective is to verify and model the pattern of radiation varying characteristics as distance and phase. During its process, different development posibilities that meet the objective of being a functional system with easy access and comprehension are analyzed. As a result, it was obtained that the system that meet the characteristics mentioned before is a uniform-linear antenna arrays with elements half-lambda dipole. Having this solution, an impendances and dephasing analysis is done with the involved elements through the Smith Chart, concluding that this system is practical as it does not require a complex design and uses daily used-elements, Compared to methods such as the Butler matrix. For the fabrication of the structure this is done with elements used daily, fulfilling the requirements initially raised.
\end{abstract}

Key words: spectrometer, antenna, impedance, adder, dipole (tesauro Ecured).

1. Artículo de investigación, enfoque cualitativo, resultado de un proceso de investigación finalizado perteneciente al área de Ingeniería Telecomunicaciones, área Antenas y Propagación de la Universidad Distrital Francisco José de Caldas. PBX: (057) (1) 3239300. Sede principal: Carrera 7 n. ${ }^{\circ}$ 40B - 53 (nueva dirección), Bogotá D. C. - (Colombia). Fecha de inicio: 1 de junio de 2016, fecha de finalización: 10 de enero de 2017.

2. Ingeniera en Telecomunicaciones, Universidad Distrital Francisco José de Caldas, Facultad de Electrónica y Telecomunicaciones, Bogotá D. C. (Colombia). Orcid: 0000-0002-5054-6576. Correo: anacarolinaramirezcleves@hotmail.com.

3. Ingeniero electrónico, Universidad Distrital Francisco José de Caldas, M. Sc. Teleinformática, Universidad Distrital Francisco José de Caldas, Profesor Facultad de Electrónica y Telecomunicaciones. Bogotá D. C. (Colombia). Orcid: 0000-0003-1694-9520. Correo: emantillab@hotmail.com. 
Un arreglo es una antena compuesta por varios elementos radiadores. Existen varios tipos de arreglos -lineales, planos, circulares, etcétera-, usados frecuentemente en el área de las telecomunicaciones. Dichos arreglos tienen como objetivo modelar las características de directividad de su radiación, haciéndolos más efectivos en el momento de transmitir y recibir su señal.

Para este proyecto se diseña un arreglo lineal de antenas dipolo, que puede ser empleado por el público en general para mejorar la calidad de la señal a recibir y transmitir. Se escogió este tipo de antena por ser sencilla y popular, ya que consiste en un hilo conductor de media longitud de onda a la frecuencia de trabajo. Como el objetivo de este proyecto es demostrar cómo modelar y verificar el patrón de radiación, este se obtiene modificando características como distancia y fase. Se analizaron las distintas posibilidades de diseño para crear arreglos de diferentes números de elementos dipolo lambda medios, y métodos como las matrices de Butler o la carta de Smith, utilizados para realizar el desfase entre cada uno de los elementos teniendo en cuenta características como la impedancia, el coeficiente de reflexión, la resistencia y la reactancia.
A continuación, se presenta un breve repaso por conceptos básicos para el diseño del mencionado arreglo, en el cual se detalla el diseño de una antena, el análisis matemático de su campo, el factor de arreglo y el uso de la carta de Smith para realizar el desfase entre los elementos radiadores. Por último, se observan el resultado y las conclusiones obtenidas, teniendo en cuenta que el sistema fue realizado en un entorno en el cual no se tiene una total aislación de ruido. Finalmente, se concluye que el arreglo realizado brinda un resultado muy aproximado al realizado en un entorno como una cámara anecoica, que es una sala diseñada para absorber en su totalidad las reflexiones producidas por ondas acústicas o electromagnéticas en cualquiera de las superficies que las conforman y con un arreglo realizado con elementos con pérdidas mínimas.

\section{Método}

\section{Tipo de estudio}

El estudio es de tipo no experimental explicativo, ya que pretende dar a comprender un fenómeno. Este tipo de estudio se concentra en la causa del evento $y$, por

Tabla 1

Especificaciones del analizador vectorial de red MS2024A

\begin{tabular}{|c|c|}
\hline Características & Descripción \\
\hline Rango de frecuencia & $2 \mathrm{MHz}$ a $4 \mathrm{GHz}$ (operacional hasta $610 \mathrm{KHz}$ ) \\
\hline Precisión de frecuencia & $25 \mathrm{ppm}$ \\
\hline Resolución de frecuencia & $10 \mathrm{~Hz}$ \\
\hline Puntos de datos & Bajo, Medio, Alto (137/275/551) \\
\hline Inmunidad a las interferencias & En el canal: $+17 \mathrm{dBm}$ \\
\hline & En frecuencia: $0 \mathrm{dBm}$ (RF Out), $+30 \mathrm{dBm}$ (RF In) \\
\hline 1-Port power & Alto: $0 \mathrm{dBm}$ (Típico) \\
\hline \multirow[t]{2}{*}{ 2-Portpower } & Alto: $0 \mathrm{dBm}$ (Típico) \\
\hline & Bajo: -35 dBm (Típico) \\
\hline Directividad corregida & $42 \mathrm{~dB}(2 \mathrm{MHz}$ to $6 \mathrm{GHz})$ \\
\hline 1-Precisión del puerto & $\begin{array}{c}=<0.44+\left|20 \log \left(1 \pm 10^{-E \Delta / 20}\right)\right| d B \text {, Típico; } E \Delta=\text { directividad }- \\
\text { Pérdida de retorno medida }\end{array}$ \\
\hline Rango dinámico & $70 \mathrm{~dB}, 2 \mathrm{MHz}$ to $10 \mathrm{MHz}$ \\
\hline & $80 \mathrm{~dB}, 10 \mathrm{MHz}$ to $3 \mathrm{GHz}$ \\
\hline & $70 \mathrm{~dB},>3 \mathrm{GHz}$ to $5.5 \mathrm{GHz}$ \\
\hline & $65 \mathrm{~dB},>5.5 \mathrm{GHz}$ to $6 \mathrm{GHz}$ \\
\hline \multirow[t]{2}{*}{ Pérdida de retorno } & Rango: 0 to $60 \mathrm{~dB}$ \\
\hline & Resolución $0.01 \mathrm{~dB}$ \\
\hline \multirow[t]{2}{*}{ VSWR } & Rango: 1 to 65 \\
\hline & Resolución 0.01 \\
\hline \multirow[t]{2}{*}{ Pérdida de cable } & Rango: 0 to $30 \mathrm{~dB}$ \\
\hline & Resolución $0.01 \mathrm{~dB}$ \\
\hline \multirow{2}{*}{ Fase de puerto } & Rango: $-180^{\circ}$ to $+180^{\circ}$ \\
\hline & Resolución: $0.01^{\circ}$ \\
\hline Carta de Smith & Resolución: 0.01 \\
\hline 2-Ganancia de puerto & Rango: -120 to $100 \mathrm{~dB}$ \\
\hline
\end{tabular}


2-Fase de puerto

Distancia a la falla
Rango: $-180^{\circ}$ to $+180^{\circ}$

Resolución: $0.01^{\circ}$

Resolución de fallos (metros): (1.5 x $108 \times \mathrm{vp}) / \Delta \mathrm{F}$; vp es la constante de propagación y $\Delta \mathrm{F}$ es $\mathrm{F} 2-\mathrm{F} 1$ en $\mathrm{Hz}$

Rango horizontal (metros): 0 a (data points-1) Resolución de fallos a un máximo de 1500m (4921ft.) donde data points $=137 / 275 / 551$

Rango vertical (pérdida de retorno): 0 to $60 \mathrm{~dB}$ Rango vertical (VSWR): 1 to 65

Fuente: Anritsu, 2008
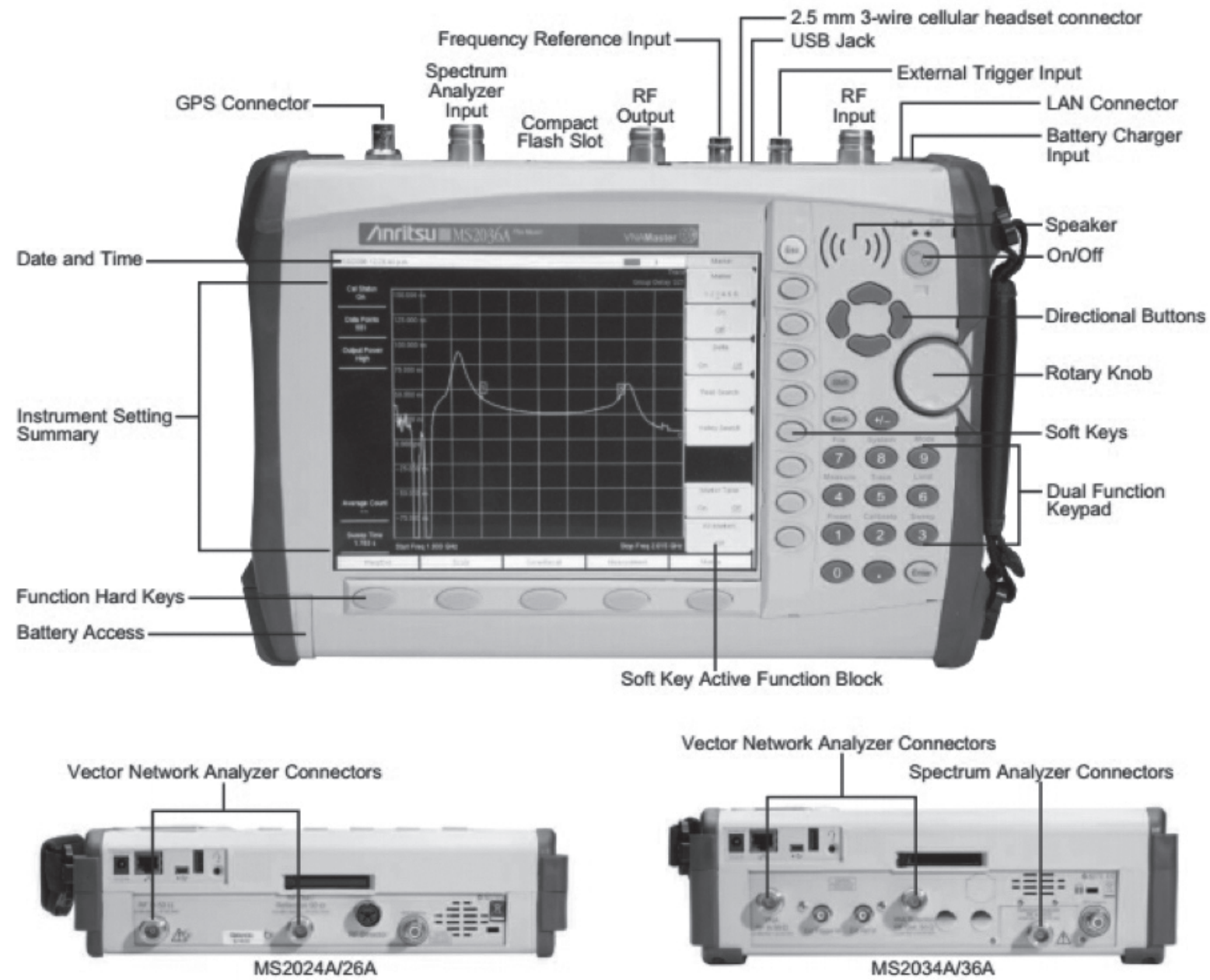

Figura 1.

VNAAnritsu MS2024A.

Fuente:Anritsu, 2008

lo tanto, está orientado a la identificación y el análisis causales y sus resultados, lo que se expresa en hechos verificables. Como se observará, el estudio se concentra en explicar el método para diseñar un arreglo de antenas lineal dipolo lambda medios, así como en verificar que dicho método cumpla con el objetivo propuesto, el cual es lograr modelar su patrón de radiación a conveniencia. 


\section{Materiales e instrumentos}

Analizador vectorial de red (VNA). El analizador vectorial de red, también llamado medidor de ganancia y fase, es tan funcional como la combinación entre un analizador de espectro y un generador de barrido. Comúnmente se encuentran de dos puertos, pero también pueden tener cuatro puertos. Su calibración es un proceso de alta precisión, en la cual se incluyen factores como la impedancia a la cual se está operando. El estándar de calibración usa cuatro dispositivos de prueba Open (red abierta), Short (red en corto circuito), Load (carga) y Thru (red conectada); los últimos dos dispositivos calibran la reflexión y la transmisión.
Analizador de espectro. Un analizador de espectro es un equipo de medición electrónico que permite visualizar en una pantalla las componentes de las señales presentes en la entrada en un espectro de frecuencias que podría ser cualquier tipo de ondas eléctricas, acústicas u ópticas. En el eje de ordenadas suele presentarse en una escala logarítmica el nivel en $\mathrm{dBm}$ del contenido espectral de la señal. En el eje de abscisas se representa la frecuencia, en una escala que es función de la separación temporal y el número de muestras captadas. Se denomina frecuencia central del analizador a la que corresponde con la frecuencia en el punto medio de la pantalla, la cual presenta el mismo comportamiento para su calibración que el analizador vectorial de espectro.

Tabla 2

Especificaciones del analizador de espectro MS2721B

\begin{tabular}{cc}
\hline Característica & Descripción \\
\hline $\begin{array}{c}\text { Frecuencia } \\
\text { Medida }\end{array}$ & $9 \mathrm{kHz}$ a $7.1 \mathrm{GHz}$ \\
Analizador de interferencias \\
Rango dinámico \\
DANL & $\begin{array}{c}\text { Ancho de banda ocupado, potencia de canal, ACPR, C/I } \\
\text { Espectrograma, intensidad de señal, RSSI } \\
\text { Ruido de fase }\end{array}$ \\
$101 \mathrm{~dB}$ en $1 \mathrm{~Hz} \mathrm{RBW}$ \\
$-163 \mathrm{dBm}$ in $1 \mathrm{~Hz} \mathrm{RBW}$ \\
Precisión de frecuencia & $-95 \mathrm{dBc} / \mathrm{Hz} @ 10 \mathrm{kHz}$ offset at $1 \mathrm{GHz}$ \\
RBW & $< \pm 25$ ppb con GPS On \\
Traza & $1 \mathrm{~Hz}$ a $3 \mathrm{MHz}$ ancho de bando de resolución \\
Detectores & normal, max hold, min hold, average, number of averages \\
Marcadores & Pico, negativo, muestra, cuasipico y verdadero RMS
\end{tabular}

Fuente: Anritsu, 2009

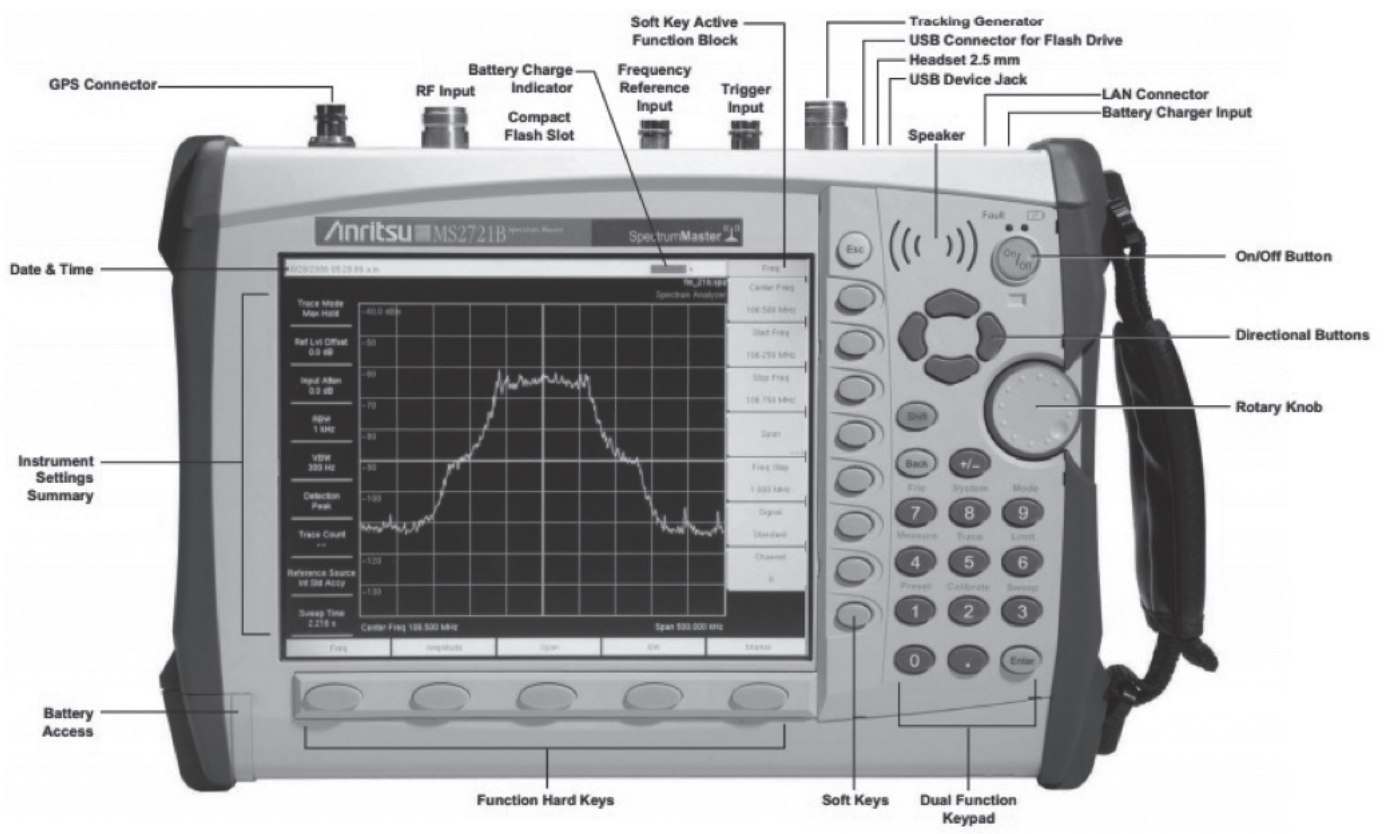

Figura 2.

Analizadordeespectro AnritsuMS2721B

Fuente:Anritsu, 2009 


\section{Marco teórico}

\section{Arreglo de antenas}

Es una antena compuesta por una cantidad de elementos radiadores ordenados de cierta manera para obtener un patrón de radiación predefinido.

Despreciando pérdidas por acoplamiento, el campo de radiación total del arreglo es la suma vectorial de los campos de los elementos radiadores.

En un arreglo de antenas es necesario tener en cuenta cinco características con el fin de modelar exitosamente el patrón de radiación:

- Tipo de configuración: geométrica, lineal, circular o plana

- Distancia de separación entre los elementos

- Amplitud de excitación de cada elemento

- Fase de excitación de cada elemento

- Patrón relativo de cada elemento

Cada uno de estos parámetros modifica las características de radiación. Considerando un arreglo de antenas lineal de dos elementos dipolo como el de la Figura 3 y su respectivo campo en la Ecuación 1.

En donde $\beta=$ diferencia de fase.

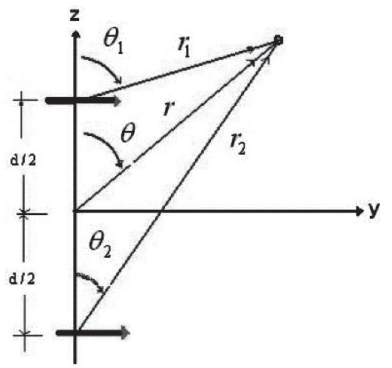

Figura 3.

Dipolo infinitesimal

Fuente: Instituto Politecnico Nacional, 2016

$\mathrm{E}_{1}=\mathrm{E}_{1}+\mathrm{E}_{2}=\hat{a}_{\theta} i \eta \frac{k I_{0} l}{4 \Pi}\left\{\frac{e^{-i\left[k r_{1}-\left(\frac{\beta}{2}\right)\right]}}{r_{1}}\left|\cos \left(\boldsymbol{\theta}_{1}\right)\right|+\frac{e^{-i\left[k r_{2}+\left(\frac{\beta}{2}\right)\right]}}{r_{2}}\left|\cos \left(\boldsymbol{\theta}_{2}\right)\right|\right\}$ (1)

Considerando el campo lejano como se muestra en la Figura 4, la magnitud de alimentación de los elementos radiadores es la misma.

Con base en la Figura 4 se tiene lo siguiente:

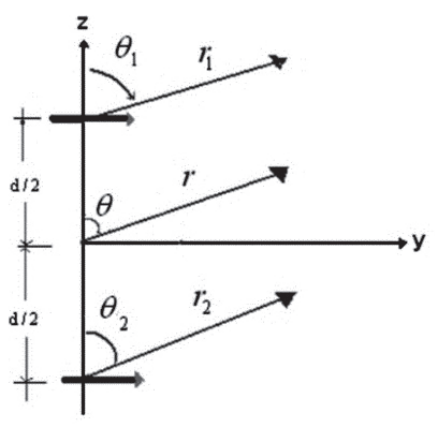

Figura 4.

Observación de un campolejano

Fuente: Instituto Politecnico Nacional, 2016

Se observa que el ángulo es el mismo, y por lo tanto las distancias de sus vectores $r$ también.

$\theta_{1} \cong \theta_{2} \cong \theta$

$\frac{r_{1} \cong r-\frac{d}{2} \cos (\theta)}{r_{2} \cong r+\frac{d}{2} \cos (\theta)}$ para variaciones en fase (3)

$r_{1} \cong r_{2} \cong r$ para variaciones en amplitud (4)

Sustituyendo en la Ecuación 1 se tiene:

$\mathrm{E}_{1}=\hat{a}_{\theta} i \eta \frac{k I_{0} l e^{-i k r}}{4 \Pi r}|\cos (\theta)|\left\{e^{+i(k d \cos \theta+\beta) / 2}+e^{-i(k d \cos \theta+\beta) / 2}\right\}$

Finalmente:

$\mathrm{E}_{1}=\hat{a}_{\theta} i \eta \frac{k I_{0} l e^{-i k r}}{4 \Pi r}|\cos (\theta)| 2 \cos \left[\frac{1}{2}(k d \cos \theta+\beta)\right](6)$

Haciendo una comparación con la Ecuación 6 indica que el campo total de un arreglo es igual al campo del elemento de origen multiplicado por un factor conocido como factor de arreglo.

$F A=2 \cos \left[\frac{1}{2}(k d \cos \theta+\beta)\right] e^{-i \alpha / 2}(7)$

$F A_{n}=2 \cos \left[\frac{1}{2}(k d \cos \theta+\beta)\right]$

\section{Carta de Smith}

La carta de Smith es una representación gráfica, en el plano del coeficiente de reflexión, de la resistencia y la reactancia normalizada. Esta herramienta permite solucionar problemas de adaptación de impedancias, que en este caso se presentan en el acople de antenas y la suma de estas; con este método se evitan las operaciones complejas que suelen implicar estos cálculos. Sobre el eje horizontal de la carta de Smith se encuentran las resistencias o las conductancias; en el centro se encuentra el valor 1, el cual, hacia la izquierda llega a 0, y hacia la derecha, va aumentando, como se muestra en la Figura 5. 


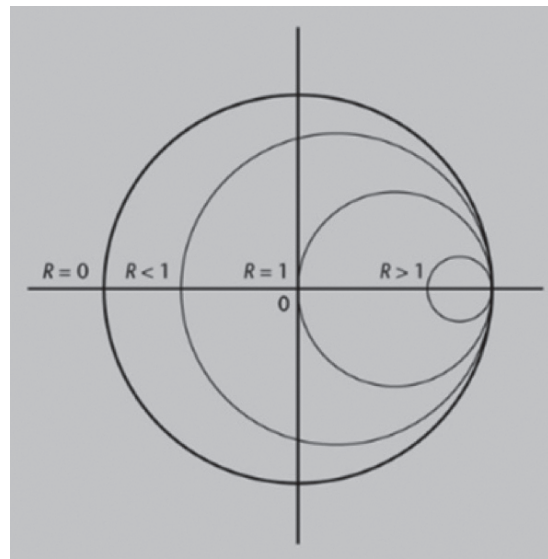

Figura 5.

Carta de Smith, valores de resistencia o conductancia

Fuente:Aleixandre, Muñoz, Atienzay Navarro, 2012

La parte reactiva de la impedancia se busca sobre los semicírculos que van desde el extremo derecho hasta algún punto del círculo extremo; si es positivo, va a la parte superior, $y$, si es negativo, hacia la inferior, como se muestra en la Figura 6.

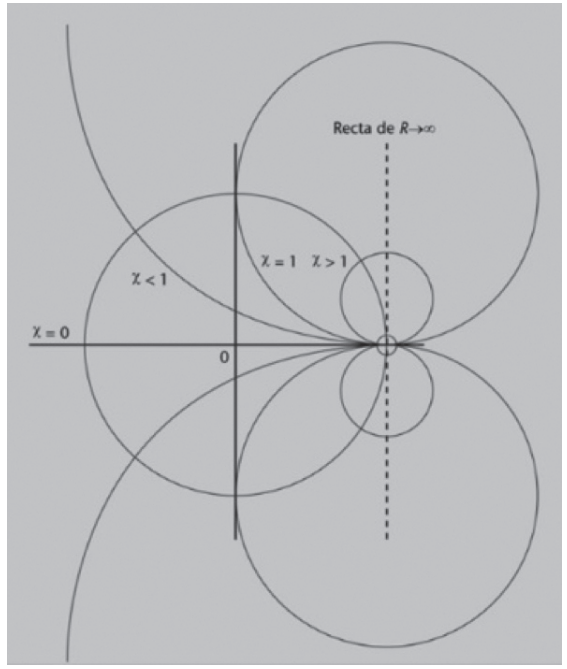

Figura 6.

Carta de Smith, valores de reactancia

Fuente:Aleixandre, Muñoz, Atienzay Navarro, 2012

Para ubicar en la carta de Smith el valor de la impedancia de la línea o elemento a analizar se debe normalizar, para ubicarlo se debe tener en cuenta la reactancia y la resistencia y poner sus valores en los círculos que se indicaron anteriormente. Ver la Figura 7

Si se extiende la línea hasta el círculo externo de la carta se debe ubicar el ángulo de fase, la magnitud es la distancia desde el centro de la carta hasta el punto ubicado anteriormente. Ver la Figura 8.

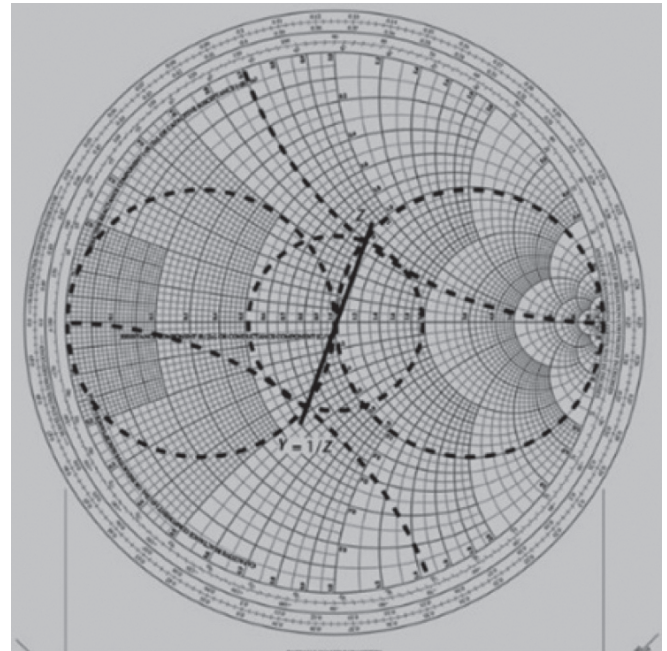

Figura 7.

Ubicación en la carta de Smith de la impedancia de la línea

Fuente:Aleixandre, Muñoz, Atienzay Navarro, 2012

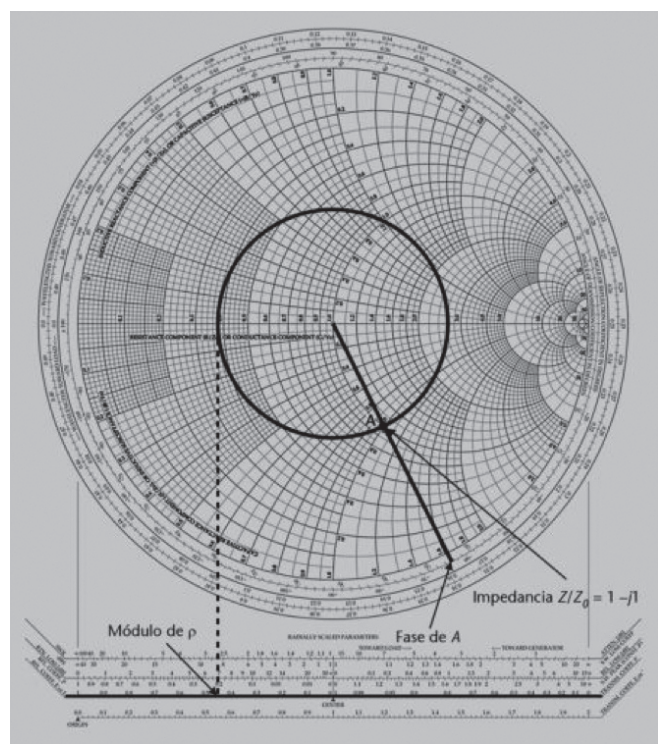

Figura 8.

Ubicación defaseymagnitud delelemento

Fuente: Aleixandre, Muñoz, Atienzay Navarro, 2012

Para realizar un desfase de la línea se debe correr el valor del ángulo; para ello se debe tener en cuenta que se puede realizar el movimiento hacia el generador o hacia la carga. Ver la Figura 9. 


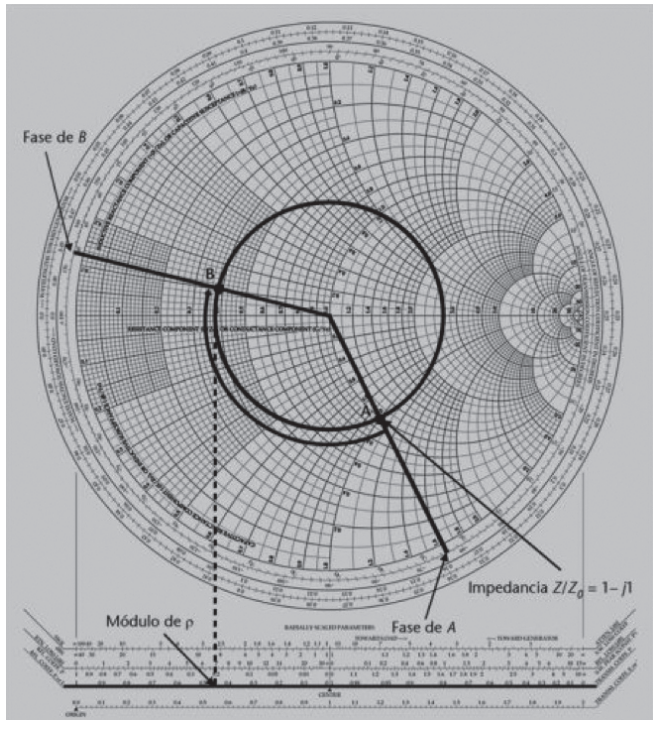

Figura 9.

Desfase delalínea

Fuente:Aleixandre, Muñoz, Atienzay Navarro, 2012

\section{Matriz de Butler}

La matriz de Butler es un dispositivo usado para diseñar amplificadores multipuerto, y especialmente, antenas inteligentes con haces de radiación conmutables.

Una matriz de Butler se realiza usando híbridos de $3 \mathrm{~dB}$, cruzadores y desfasadores. Su ancho de banda y sus pérdidas vendrán determinados por el diseño de estos elementos.

Un acoplador es un dispositivo pasivo comúnmente de cuatro puertos, cuyo funcionamiento se basa en dividir la potencia de la señal insertada en un puerto entre sus salidas, de forma simétrica o asimétrica, dependiendo de las características del acoplador.

En el interior de la matriz de Butler es necesario cruzar físicamente dos señales sin que se afecten sus propiedades electromagnéticas y se mantenga un alto aislamiento. Para realizar esta tarea se usa un cruzador.

Las propiedades básicas de una matriz de Butler son: el aislamiento entre cada una de sus entradas, la linealidad en fase con respecto a la posición de salida y el incremento de fase dependiendo de la entrada seleccionada.

Matemáticamente, la matriz de Butler realiza una transformada rápida de Fourier, generando lóbulos que son ortogonales entre sí. La disposición espacial que tendrán los lóbulos depende de las características con las que se diseñen los elementos que conforman la matriz (Danilo, 2016).
Se descartó el uso de la matriz de Butler debido a su complejidad, ya que uno de los objetivos requeridos es que sea un sistema funcional, de fácil acceso al público y de fácil comprensión. Adicionalmente, su sistema se implementa sobre un circuito impreso y con antenas de microcinta.

\section{Estructura}

- Materiales 1 tubo PVC de $1 / 2 "$ de 1 metro

- 2 tubos PVC de $1 / 2$ " de 2 metros

- 5 uniones en T de PVC de $1 / 2$ "

- 4 uniones en T de PVC de 3/8"

- 1 base de silla giratoria

- 8 tubos de aluminio de $3 / 8^{\prime \prime}$ de $24,1925 \mathrm{~cm}$.

- 5 conectores SMA macho para cable coaxial

- 6 metros de cable coaxial

- 1 sumador channel plus 2534

- 4 tubos de aluminio o de madera de $20 \mathrm{~cm}$

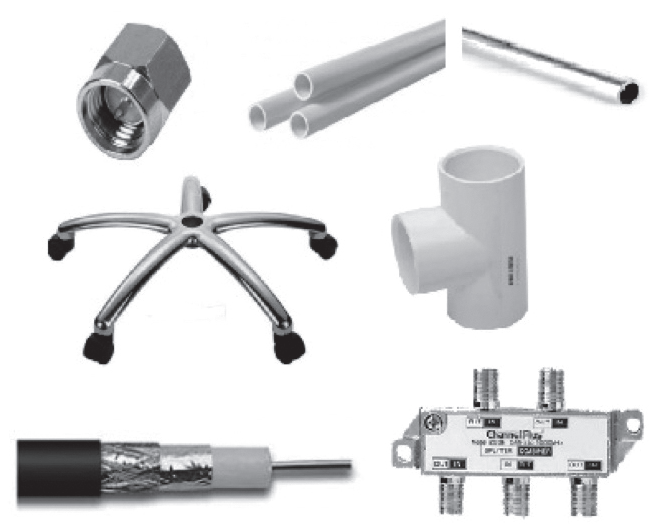

Figura 10.

Materiales

Fuente:Autores

\section{Dipolo}

En cada extremo de la $T$ de $3 / 8^{\prime \prime}$ se deben introducir las varillas correspondientes - de $24,1925 \mathrm{~cm}$-, estas no deben unirse y deben fijarse a cada extremo con un tornillo que atraviese la T por completo; de igual manera, por el centro de la T se debe introducir el cable coaxial y conectarse a los tornillos ubicados en cada extremo; es importante tener en cuenta que el cable coaxial tiene un alambre en el centro y un recubrimiento de aluminio alrededor que deben conectarse a los extremos indicados anteriormente. Para conocer la medida del cable se debe tener en cuenta el punto 3 del procedimiento descrito más adelante; es recomendable, además, que se fije en el centro con silicona (esto con el fin de evitar el contacto); al final del cable coaxial se debe introducir el 
conector SMA macho. Adicionalmente, se debe colocar un soporte, que puede ser un tubo de aluminio o una vara de madera (cuya longitud puede ser de $20 \mathrm{~cm}$ ) para acoplar los dipolos en la estructura base (ver figura 11).

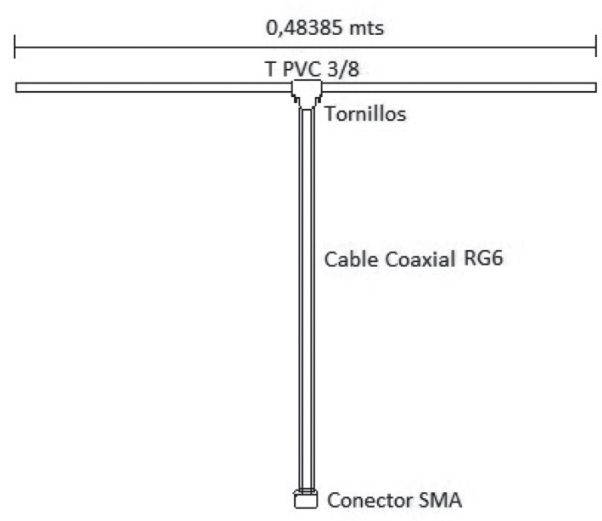

Figura 11.

Construcción del dipolo

Fuente:Autores

\section{Base}

El tubo de PVC se debe conectar a la silla giratoria, en cuyo centro se pone una hoja de papel que tiene impreso un círculo indicando los grados respectivos $\left(0^{\circ}\right.$ a $\left.360^{\circ}\right)$; en el extremo superior se debe introducir una T de $1 / 2$ " $y$ en cada extremo de esta, un tubo de PVC de un metro; sobre cada uno de estos tubos hay que insertar dos T de $1 / 2$ " para poder acoplar cada uno de los dipolos (ver Figura $12)$.

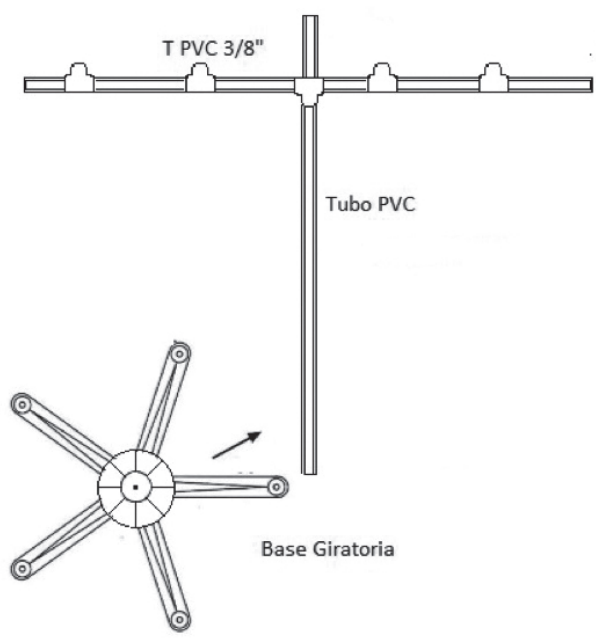

Figura 12.

Construcción base de arreglo

Fuente:Autores

\section{Conexión final}

Para realizar la medición del arreglo se deben colocar los dipolos correspondientes en una de las cuatro T de PVC ubicadas en la estructura base; el conector SMA de cada uno de los dipolos debe conectarse al sumador 2534 y la salida de este debe conectarse al VNA (ver Figura 13).

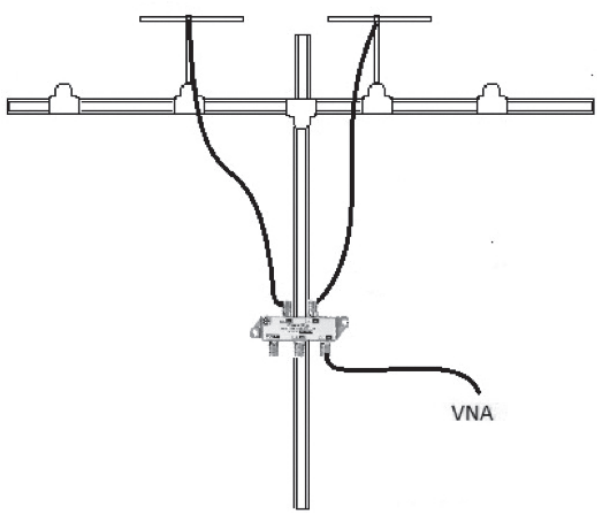

Figura 13.

Conexión final del arreglo

Fuente:Autores

\section{Procedimiento}

El proceso para realizar un arreglo de antenas lineal es el siguiente:

1. Según el análisis teórico de un arreglo lineal de antenas que se realizó previamente, se decidió realizar cinco dipolos $\lambda / 2$ a una frecuencia de $310 \mathrm{MHz}$ (ver Figura 14).

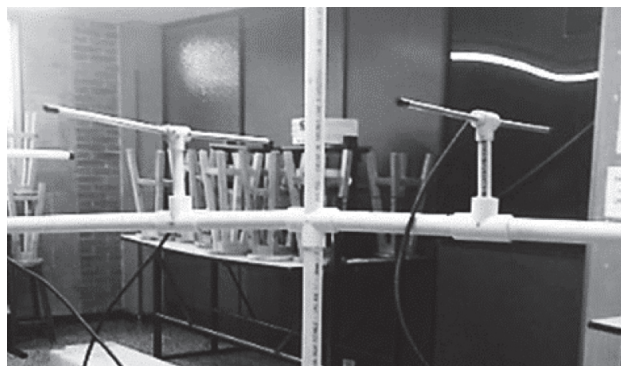

Figura 14.

Dipolos $\lambda / 2$ sobre estructura

Fuente:Autores

2. Con el fin de saber cuál es su impedancia y así ubicarlas en la carta de Smith, se mide cada una de las antenas en el VNA previamente calibrado. 


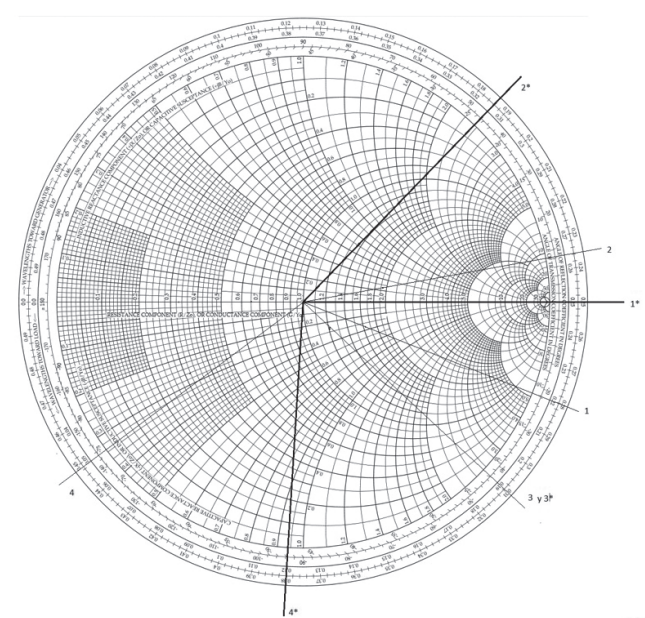

Figura 15.

Ubicación de impedancias de antenasysu desfase en la carta de Smith Fuente:Autores

3. Después de obtener las impedancias se debe desfasar a $45^{\circ}$, por lo cual se movió hacia el generador la impedancia; para hacerlo se cortó la línea de transmisión. Para saber cuánto se debe cortar, la parte exterior de la carta de Smith indica a cuantas lambdas está la impedancia del generador. En la Figura 15 se observa que las líneas negras oscuras son las líneas ubicadas ya desfasadas, y la línea de referencia es la 3. Luego, se hace la conversión de lambdas a centímetros con el fin de realizar el corte del cable; para ello se debe tener en cuenta la Ecuación 9.

$\lambda=\frac{c}{f}=\frac{3 * 10^{8}}{310 * 10^{6}}=0.9677 \mathrm{~m}(9)$

4. Después de que el elemento se encuentra desfasado y cada uno es ubicado, se suman los dipolos; para ello se utilizó un sumador Channel plus 2534 como el que se observa en la Figura 16.

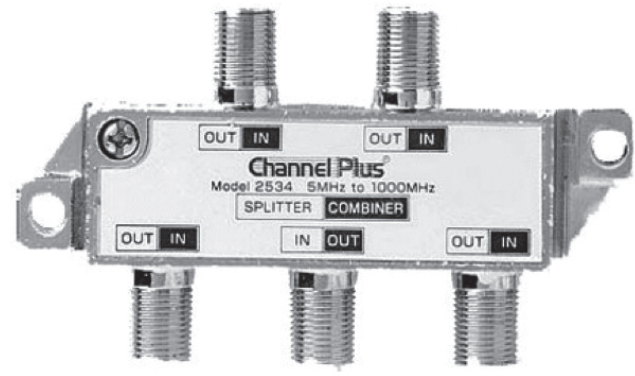

Figura 16.

Sumadorchannel plus 2534

Fuente:Solid Signal, Signal Group LLC \& Affiliates, 2002

- $1 \mathrm{GHz}$ bandwidth

- $\quad 9 \mathrm{~dB}$ insertion loss

5. Después, se usa el analizador de espectro (previamente calibrado) para verificar la potencia en cada punto. En la base de la estructura hay un marcador de $360^{\circ}$, que es la guía para girar la estructura cada $5^{\circ}$. Con el fin de construir la gráfica de patrón de radiación -que se observa en la Figura 17- se debe tomar cada potencia y ángulo como se muestra en la Tabla 3.

Tabla 3

Muestra de recopilación de datos

\begin{tabular}{cc}
\hline Ángulo & Potencia \\
\hline 0 & 0,014663484 \\
5 & 0,010544153 \\
10 & 0,010057279 \\
15 & 0,008221957 \\
20 & 0,030176611 \\
25 & 0,051615752 \\
30 & 0,106078759 \\
35 & 0,149193317 \\
40 & 0,250596659 \\
45 & 0,319809069 \\
\hline
\end{tabular}

Fuente: Autores

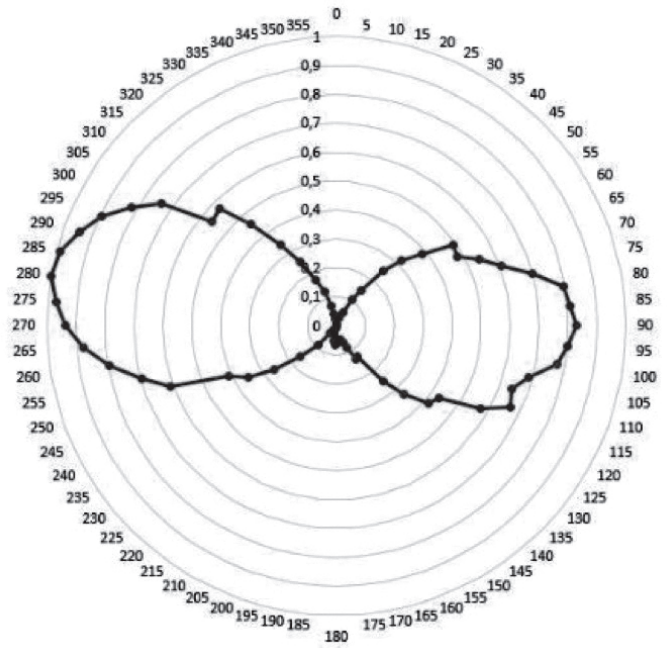

Figura 17.

Patrón de radiación resultante

Fuente:Autores

Se da crédito a los estudiantes de la Universidad Distrital, quienes diseñaron la estructura donde se ubicaron las antenas para realizar el laboratorio, permitiendo así medir el patrón de radiación.

\section{Diagrama de conexión}

Este diagrama muestra la conexión mencionada en el procedimiento descrito en el índice anterior (ver Figura 18). 


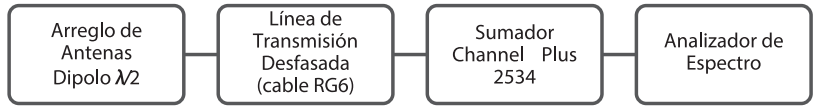

Figura 18.

Diagrama de conexión del arreglo lineal de antenas dipoloN/2

Fuente: Autores

\section{Análisis matemático}

Desfase de línea de transmisión. Según el punto 2 del procedimiento, se debe medir la impedancia de cada una de las antenas, las cuales arrojaron los siguientes resultados:

Tabla 4

Impedancias de las antenas

\begin{tabular}{cc}
\hline Ángulo & Impedancia Z \\
\hline Antena 1 & $122,8-27,64 \mathrm{i}$ \\
Antena 2 & $145,02+21,04 \mathrm{i}$ \\
Antena 3 & $91,86-20,37 \mathrm{i}$ \\
Antena 4 & $36,12-20,81 \mathrm{i}$ \\
\hline
\end{tabular}

\section{Fuente: Autores}

Primero se debe normalizar respecto a la impedancia característica de la línea de transmisión. Se sabe que la impedancia característica de la línea es $75 \Omega$ ya que los cables son de calibre RG6 y tienen una impedancia de $75 \Omega$, como también las entradas de los elementos como el sumador.

Para normalizar se aplica la Ecuación 10.

$$
|Z|=\frac{Z}{Z_{0}}(10)
$$

Normalizando, el resultado de las impedancias es el siguiente (ver Tabla 5):

\section{Tabla 5}

Impedancias normalizadas

\begin{tabular}{cc}
\hline Ángulo & Impedancia $|\mathrm{Z}|$ \\
\hline Antena 1 & $1,63733-0,368533 \mathrm{i}$ \\
Antena 2 & $1,9336+0,280533 \mathrm{i}$ \\
Antena 3 & $1,2248-0,2716 \mathrm{i}$ \\
Antena 4 & $0,4816-0,277467 \mathrm{i}$ \\
\hline
\end{tabular}

\section{Fuente: Autores}

Para realizar el desfase es necesario hallar el coeficiente de reflexión, el cual es el parámetro que indica qué porcentaje (o porción) de la onda incidente no es consumida o utilizada por la carga y es devuelta a la fuente en forma de onda regresiva; para hallarla se utiliza la Ecuación 11:

$\rho=\frac{|Z|-1}{|Z|+1}(11)$

Los coeficientes de reflexión de las antenas son los siguientes (ver Tabla 6):

Tabla 6

\section{Coeficientes de reflexión}

\begin{tabular}{lcc}
\hline Antena & $\begin{array}{c}\text { Coeficiente de } \\
\text { reflexión } \mathbf{p}\end{array}$ & $\begin{array}{c}\text { Coeficiente de } \\
\text { reflexión } \boldsymbol{\rho}\end{array}$ \\
\hline Antena 1 & $0,256182-0,10393 \mathrm{i}$ & $0,276465 \mathrm{t}-22,0835 \circ 0,2764651337,9165$ \\
Antena 2 & $0,324422+0.064604 \mathrm{i}$ & $0,330792111,2623$ \\
Antena 3 & $0,114243-0,10813 \mathrm{i}$ & $0,1573021-43,4257 \circ 0,1573021316,5743$ \\
Antena 4 & $-0,304153-0,2442 \mathrm{i}$ & $0,390077 \mathrm{t}-141,235 \circ 0,3900771218,765$
\end{tabular}

\section{Fuente: Autores}

El objetivo es lograr desfasar la antena con respecto a la inmediatamente siguiente en $45^{\circ}$, para esto se deben analizar los cuatro ángulos (ver Tabla 7) y así correr la línea lo menor posible con el fin de no acortar en exceso la longitud del cable. Es recomendable, además, verificar el valor de la distancia entre las antenas.

\section{Tabla 7}

Ángulo de distancia / Diferencia entre antenas

\begin{tabular}{cc}
\hline Antenas & $\begin{array}{c}\text { Ángulo de distancia / } \\
\text { Diferencia entre antenas }\end{array}$ \\
\hline 1 y 2 & $326.654^{\circ}$ \\
2 y 3 & $305.312^{\circ}$ \\
3 y 4 & $97,8093^{\circ}$ \\
4 y 1 & $119.152^{\circ}$ \\
1 y 3 & $21.3422^{\circ}$ \\
2 y 4 & $207.503^{\circ}$ \\
\hline
\end{tabular}

Fuente: Autores

Con los resultados obtenidos anteriormente, se debe escoger una antena que sea referencia con respecto a las demás para realizar los desfases; es recomendable graficarlas para así encontrar una solución óptima que satisfaga el desarrollo del desfase.

Como se puede observar en la Figura 15, se deduce la siguiente solución:

Se debe correr la fase en dirección hacia la carga, la antena 4 corre hacia la antena 3, la antena 1 corre hacia la antena 2, esto para obtener un desfase de $45^{\circ}$ entre la antena 3 y la antena 1 ; y por último, la antena 2 con 
respecto a la antena 3 debe tener $90^{\circ}$ de desfase, con esto se debe cumplir el requerimiento de que estén a $45^{\circ}$ con respecto a la inmediatamente siguiente.

Desfase antena 4

$\emptyset_{4}=\emptyset_{3}-45^{\circ}=316,5743^{\circ}-45^{\circ}=271,5743$

El nuevo ángulo de la antena 4 es $\emptyset_{4}=271,5743^{\circ}$

Desfase antena 1

$\emptyset_{1}=\emptyset_{3}+45^{\circ}=316,5743^{\circ}+45^{\circ}=361,5743$

Como ya se dio una vuelta de $360^{\circ}$, se resta esta para verificar el ángulo real desde el plano 0 o inicial.

El nuevo ángulo de la antena 1 es $\emptyset_{1}=1,5743^{\circ}$

Desfase antena 2

$\emptyset_{2}=\emptyset_{3}+90^{\circ}=316,5743^{\circ}+90^{\circ}=406,5743$

Como ya se dio una vuelta de $360^{\circ}$ se resta esta para verificar el ángulo real desde el plano 0 o inicial.

El nuevo ángulo de la antena 2 es $\emptyset_{2}=46,5743^{\circ}$

Los valores del coeficiente de reflexión con desfase a $45^{\circ}$ se muestran en la Tabla 8:

Tabla 8

Valores del coeficiente de reflexión con desfase a $45^{\circ}$

\begin{tabular}{cc}
\hline Antenas & $\begin{array}{c}\text { Coeficiente de } \\
\text { reflexión } \boldsymbol{\rho}\end{array}$ \\
\hline 1 & $0,27646511,5743^{\circ}$ \\
2 & $0,330792146,5743$ \\
3 & $0,1573021-43,4257$ \\
4 & $0,3900771-88,4257$ \\
\hline
\end{tabular}

\section{Fuente: Autores}

El corrimiento de las líneas de transmisión se puede verificar en la carta de Smith. Ahora se debe hallar las nuevas impedancias normalizadas |Z| con la Ecuación 15 (ver Tabla 9).

$|Z|=\frac{-1-\rho}{\rho-1}$

Con el fin de conocer la distancia final del cable coaxial para obtener el desfase requerido, se debe realizar la conversión de lambdas al sistema métrico decimal (centímetros o metros). Para ello se debe tener en cuenta la Ecuación 9.
Tabla 9 Impedancias después de análisis de desfase $45^{\circ}$

\begin{tabular}{cc}
\hline Antena & Nuevas impedancia $|\mathrm{Z}|$ \\
\hline Antena 1 & $1.7635+0.029006 \mathrm{i}$ \\
Antena 2 & $1.3604+0.7339 \mathrm{i}$ \\
Antena 3 & $1,2248-0,2716 \mathrm{i}$ \\
Antena 4 & $0.749819-0.689698 \mathrm{i}$ \\
\hline
\end{tabular}

Fuente: los autores

En la carta de Smith hay una sección que está en la parte exterior de la circunferencia, como se había mencionado anteriormente, esto indica una métrica hacia el generador y hacia la carga en una escala de 0.1. En la Figura 19 se puede ver la representación de la impedancia original y la que contiene el desfase; extendiendo la línea se observa que esta se cruza con un valor en la parte exterior de la circunferencia.

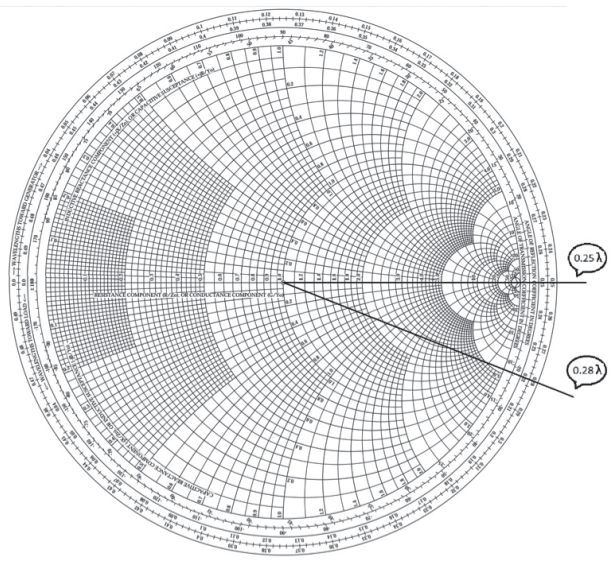

Figura 19.

Representación de la antena 1 métrica de lambda Fuente:Autores

A continuación, se restan dichos valores y este resultado se convierte de lambda a centímetros o metros mediante regla de tres.

$(0.28-0.25) \lambda=0.029 \lambda(16)$
$\frac{0.029 \lambda * 0.9677 \mathrm{~m}}{\lambda}=0.02806 \mathrm{~m}=2.8 \mathrm{~cm} \quad(17)$

Teniendo en cuenta que la medida del cable es de aproximadamente un metro, a la antena 1 se deben cortar $2.8 \mathrm{~cm}$ para obtener dicho desfase en la línea; este mismo proceso se realiza con la antena 2 . Como la antena 3 fue la base para realizar el desfase no tiene ningún cambio. Por último-como se observa en la Figura 15-, la antena 4 tiene que realizar un giro completo y se tendría 
que hacer un corte muy grande al cable si este fuera de un metro, por lo cual este se cortó más largo y se tomó hacia la carga; sin embargo, se realiza el mismo proceso anterior teniendo en cuenta que el valor obtenido es la adición de cable.

Teniendo en cuenta que la medida del cable es de aproximadamente un metro, a la antena 1 se deben cortar $2.8 \mathrm{~cm}$ para obtener dicho desfase en la línea; este mismo proceso se realiza con la antena 2. Como la antena 3 fue la base para realizar el desfase no tiene ningún cambio. Por último-como se observa en la Figura 15-, la antena 4 tiene que realizar un giro completo y se tendría que hacer un corte muy grande al cable si este fuera de un metro, por lo cual este se cortó más largo y se tomó hacia la carga; sin embargo, se realiza el mismo proceso anterior teniendo en cuenta que el valor obtenido es la adición de cable.

$(0.444-0.374) \lambda=0.07 \lambda$

$\frac{0.07 \lambda * 0.9677 \mathrm{~m}}{\lambda}=0.0677396 \mathrm{~m}=6,7 \mathrm{~cm}(19)$

Patrón de radiación. Se debe tener en cuenta que durante el proyecto se realizaron varias medidas con los dipolos lambda medios a distancias de lambda medios, lambda tercios y lambda, con ángulos de desfase de $45^{\circ}$, $90^{\circ}$ y $135^{\circ}$ aproximadamente.

Se sabe que la potencia de un dipolo lambda medios es:

$P t x=\left(\frac{\left(\cos \left(\frac{\beta \cdot L}{2} \cdot \cos (\theta)\right)-\cos \left(\frac{\beta \cdot L}{2}\right)\right)}{\sin (\theta)}\right)^{2}$

Y el factor de arreglo es:

$F A=\left(\operatorname{Cos}\left(\frac{1}{2}(\beta * d * \operatorname{Cos}(\theta))+\alpha\right)\right)^{2}$

La potencia total de un arreglo de dos antenas es igual:

Ptotal arreglo $=$ Ptx $*$ FA (22)

Este procedimiento indica que se debe hacer un barrido y tomar valores cada $5^{\circ}$ grados; de manera teórica, tomando como ejemplo un ángulo de barrido de $45^{\circ}$ de un dipolo lambda medios a una distancia de lambda medios y un desfase entre dipolos de $45^{\circ}$, esto se calcularía de la siguiente manera. Cabe resaltar que es mejor realizar dichos cálculos en un programa matemático como Matlab o Microsoft Excel (ya que es necesario realizar un barrido o listado de ángulos), por lo cual se debe hacer el cambio de grados a radianes. $\lambda=\frac{c}{f}=\frac{3 * 10^{8}}{310 * 10^{6}}=0,967741 \mathrm{~cm}(23)$

$\beta=\frac{2 * \pi}{\lambda}=6,4926$

Distancia entre los elementos $d=\frac{\lambda}{2}=0,48387097$ (25)

Ángulo de desfase entre los elementos

$\alpha=\frac{45 * \pi}{180}=0,785398 \quad(26)$

Ángulo de barrido $\theta=\frac{45 * \pi}{180}=0,785398 \quad$ (27)

$$
\begin{aligned}
P t x & =\left(\frac{(\cos (1,570796 * \operatorname{Cos}(0,785398))-\operatorname{Cos}(1,570796))}{\operatorname{Sin}(0,785398)}\right)^{2} \\
& =0,394300133 \quad(28)
\end{aligned}
$$

$F A=\left(\operatorname{Cos}\left(\frac{1}{2}(6,4926 * 0,48387097 * \operatorname{Cos}(0,785398))\right.\right.$

$$
+0,785398))^{2}=0,00178726
$$

Ptotal arreglo $=0,394300133 * 0,004532729=0,00178726$ (30)

Después de hacer dichos cálculos con cada uno de los ángulos correspondientes desde $0^{\circ}$ a $360^{\circ}$, se puede realizar una gráfica para verificar su respectivo patrón de radiación, como se muestra en la Figura 20. 


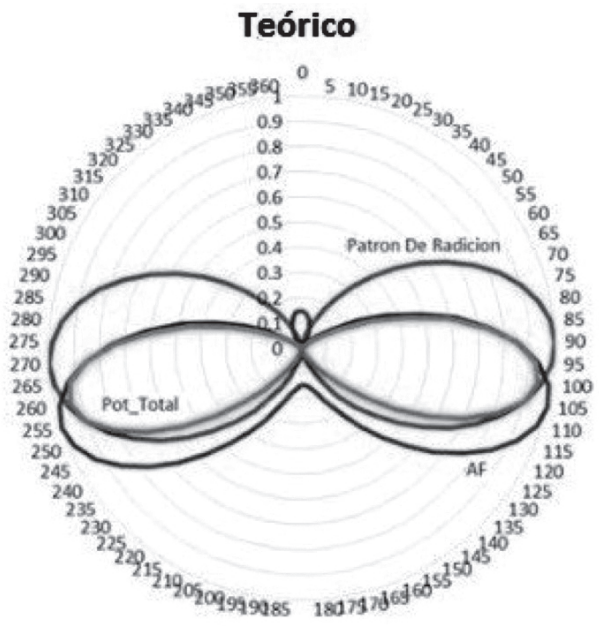

Figura 20.

Resultados teóricos/prácticos arreglo de dos dipolos $N 2$, distancia $N 2$ desfase $45^{\circ}$ Fuente:Autores

Los resultados obtenidos pueden ser comparados con el programa Amanogawa, el cual brinda una visión del resultado del patrón de radiación resultante; sin embargo, se debe tener en cuenta que este brinda la información del factor de arreglo y la del campo por separado (ver Figura 21).

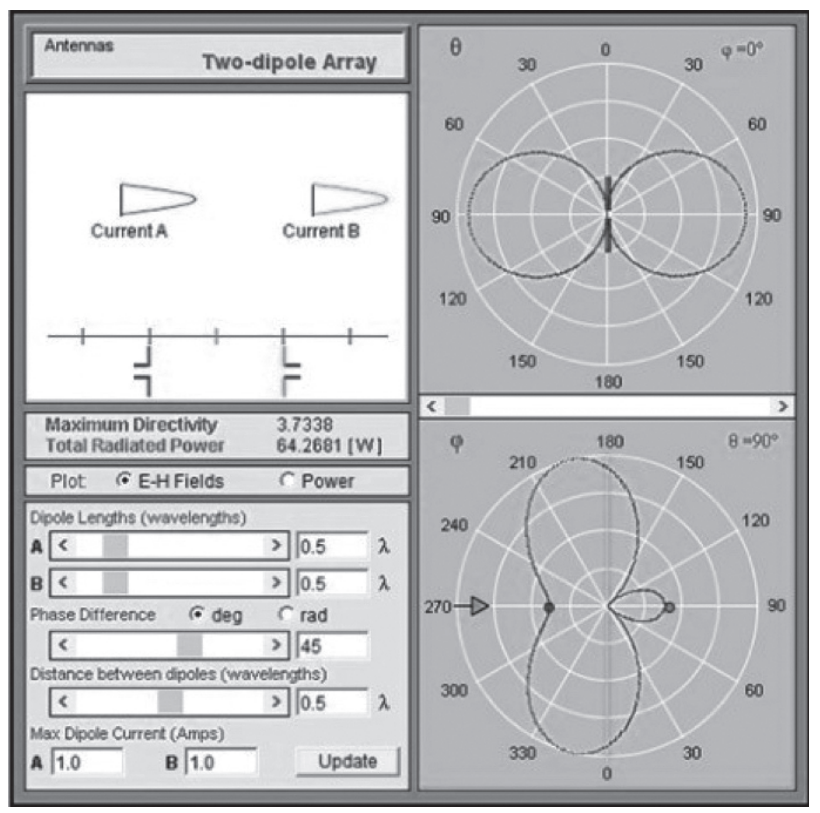

Figura 21.

Resultados Amanogawa arreglo 2 Antenas dipoloN2, distancia N2, desfase $45^{\circ}$. Fuente:SEMCHIPSemchip(s.f.)

También se realizó la simulación de los datos teóricos en $3 \mathrm{D}$ en el programa Matcad, con el fin de tener una perspectiva diferente del patrón de radiación de cada elemento y del arreglo (ver Figuras 22-30).

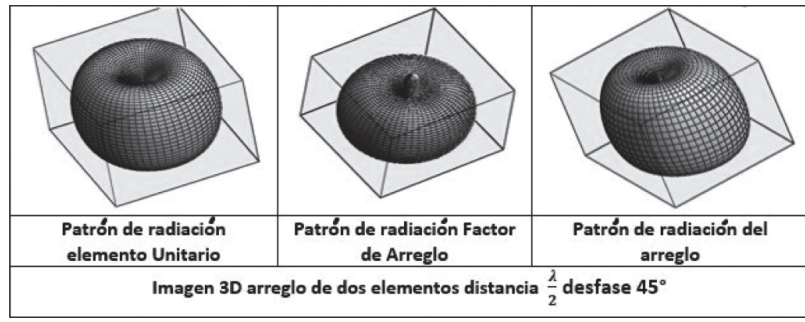

Figura 22.

Simulación 3D Matcad. Arreglo 2 antenas dipolo N2, distancia N2, desfase $45^{\circ}$ Fuente: Autores

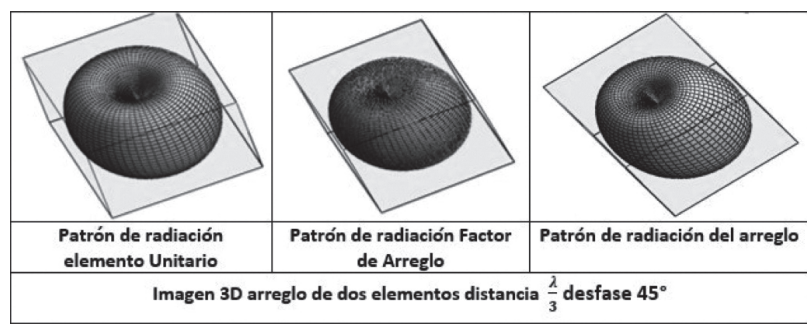

Figura 23.

Simulación 3D Matcad. Arreglo 2 antenas dipolo N2, distancia N3, desfase $45^{\circ}$ Fuente: Autores

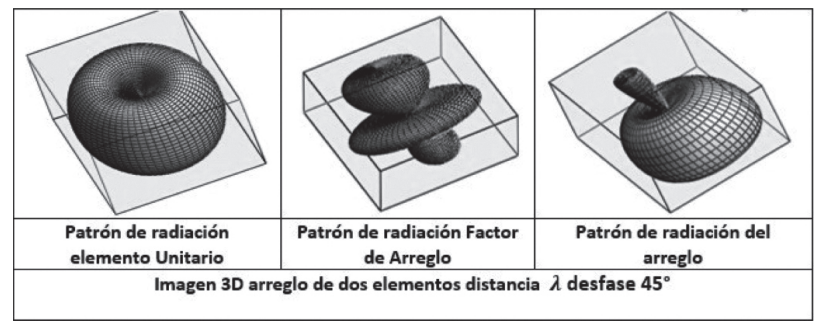

Figura 24.

Simulación 3D Matcad. Arreglo 2 antenas dipolo N2, distancia $\lambda$, desfase $45^{\circ}$ Fuente:Autores

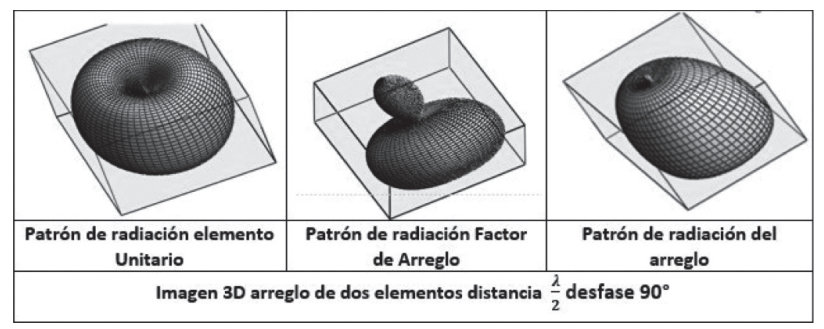

Figura 25.

Simulación 3D Matcad. Arreglo 2 antenas dipolo N2, distancia N2, desfase $90^{\circ}$ Fuente:Autores 


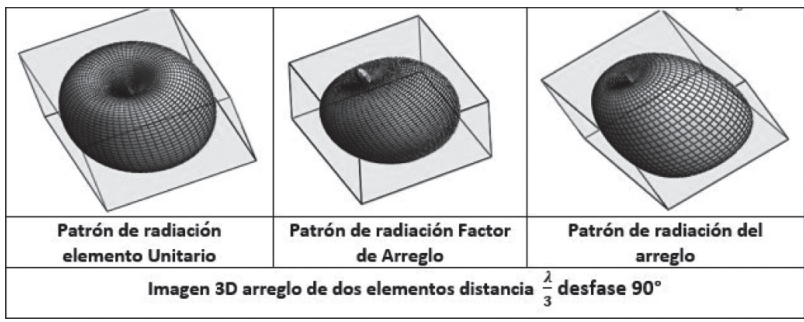

Figura 26.

Simulación 3D Matcad. Arreglo 2 antenas dipolo N2, distancia N3, desfase $90^{\circ}$ Fuente:Autores

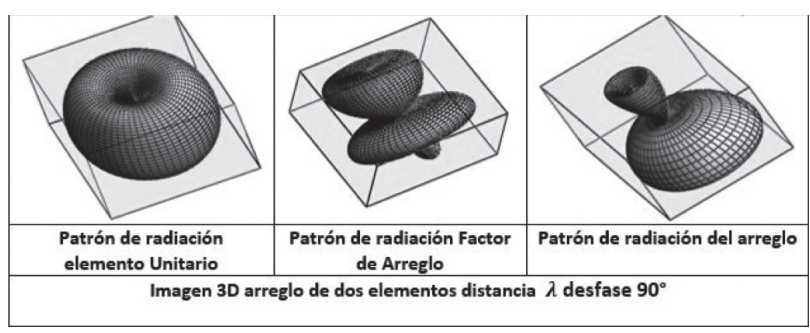

Figura 27.

Simulación 3D Matcad. Arreglo 2 antenas dipolo N2, distancia $\lambda$, desfase $90^{\circ}$ Fuente:Autores

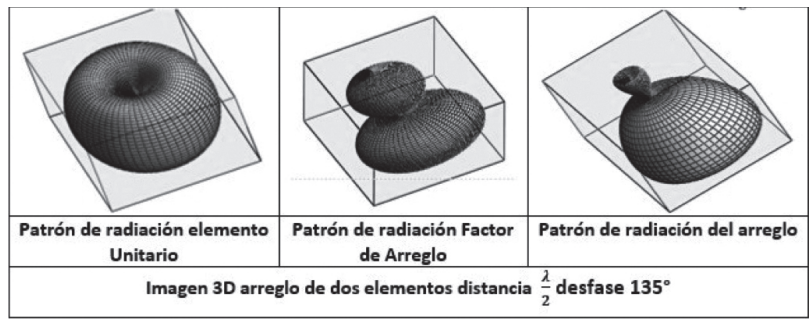

Figura 28.

Simulación 3D Matcad. Arreglo 2 antenas dipolo N2, distancia N2, desfase $135^{\circ}$ Fuente:Autores

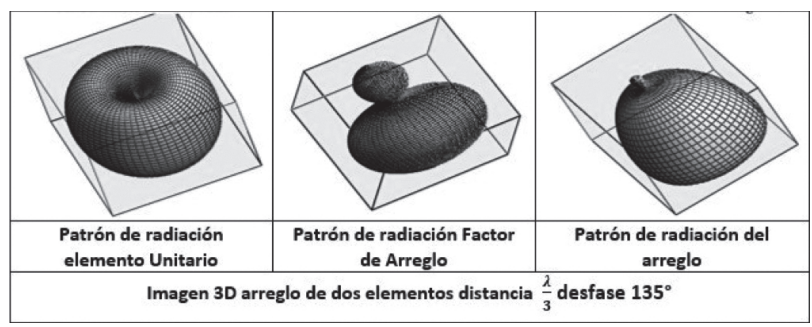

Figura 29.

Simulación 3D Matcad. Arreglo 2 antenas dipolo N2, distancia N3, desfase $135^{\circ}$. Fuente:Autores

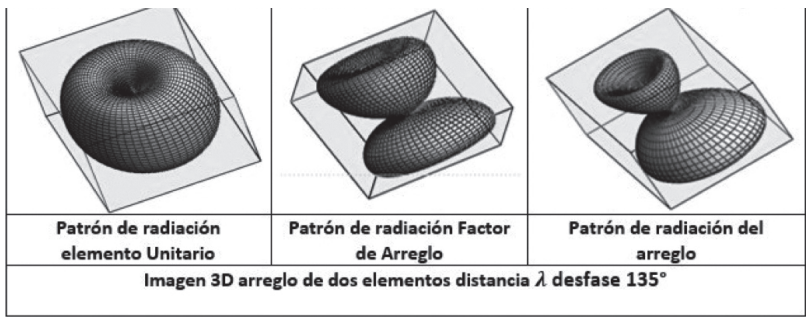

Figura 30.

Simulación 3D Matcad. Arreglo 2 antenas dipolo N2, distancia $\lambda$, desfase $135^{\circ}$ Fuente:Autores

\section{Resultados}

A continuación, en las Tablas 10, 11 y 12, se muestran las gráficas de los resultados obtenidos con las fórmulas teóricas empleadas teniendo en cuenta su desfase, la distancia y la cantidad de elementos (columna derecha); también se observan las gráficas de los resultados obtenidos de las mediciones realizadas en el analizador de espectro (columna izquierda). En estas gráficas es posible observar que los datos teóricos y prácticos son aproximados, esto teniendo en cuenta que el entorno en el cual se ejecutó el arreglo presentaba ruido y no se encontraba aislada de ondas electromagnéticas o acústicas, como ocurriría en una cámara anecoica. En las gráficas teóricas se incluyó el campo de un solo elemento dipolo y el factor de arreglo para poder observar y diferenciar el resultado de su multiplicación, el cual es el campo resultante del arreglo. 


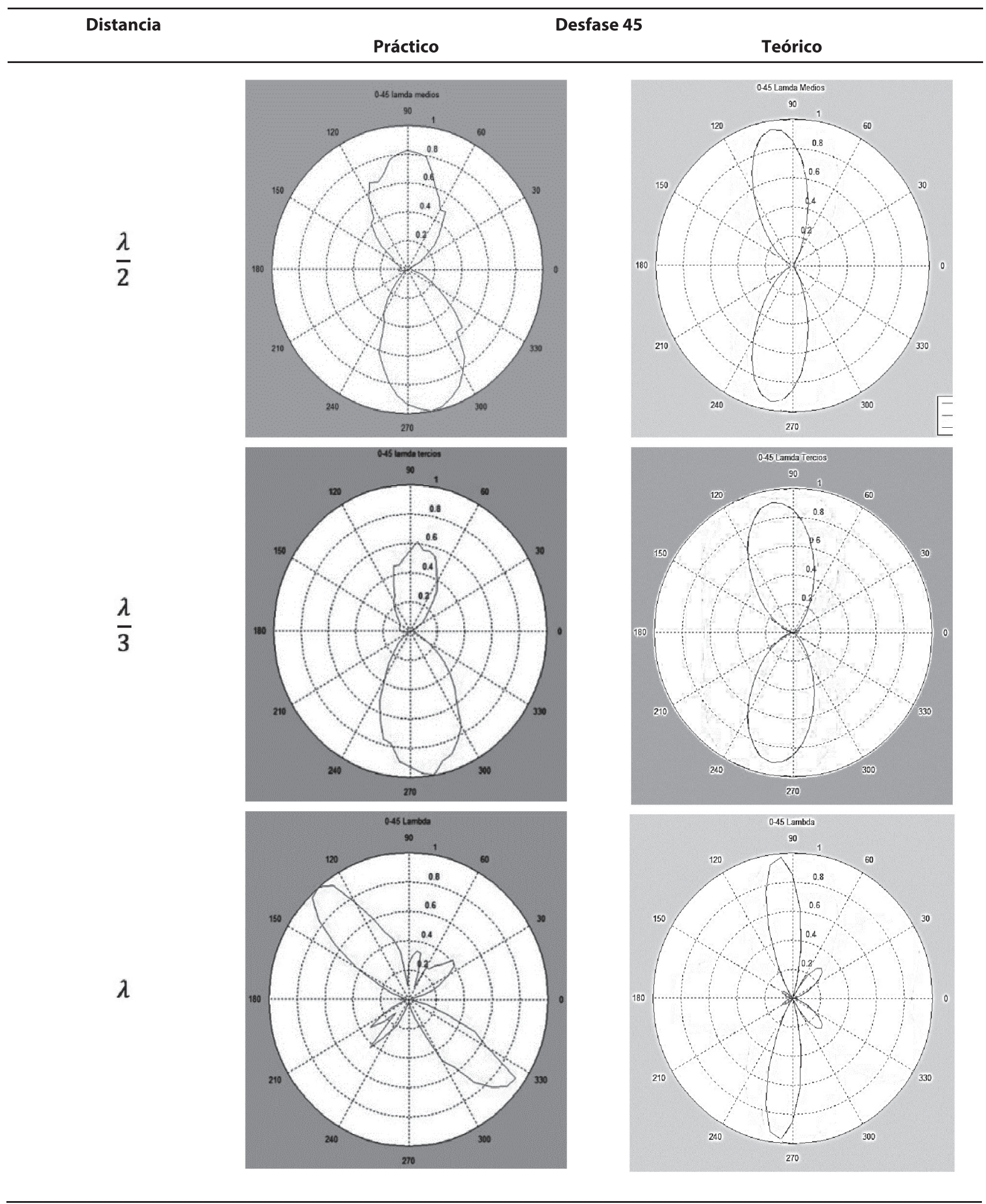

Fuente: Autores 
Tabla 11

Resultados teóricos y prácticos del desfase de $90^{\circ}$

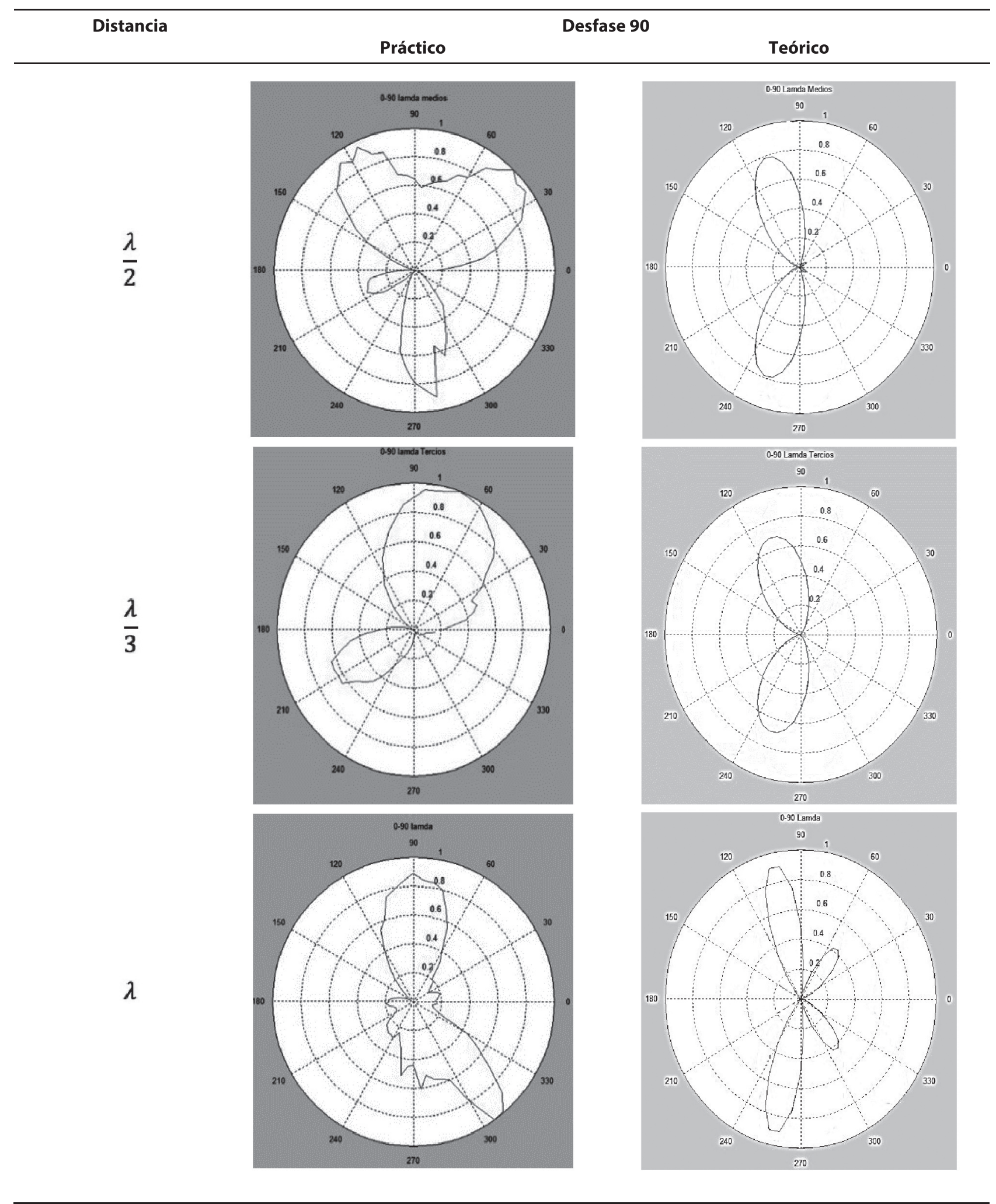

Fuente: Autores 
Tabla 12

Resultados teóricos y prácticos del desfase de 135

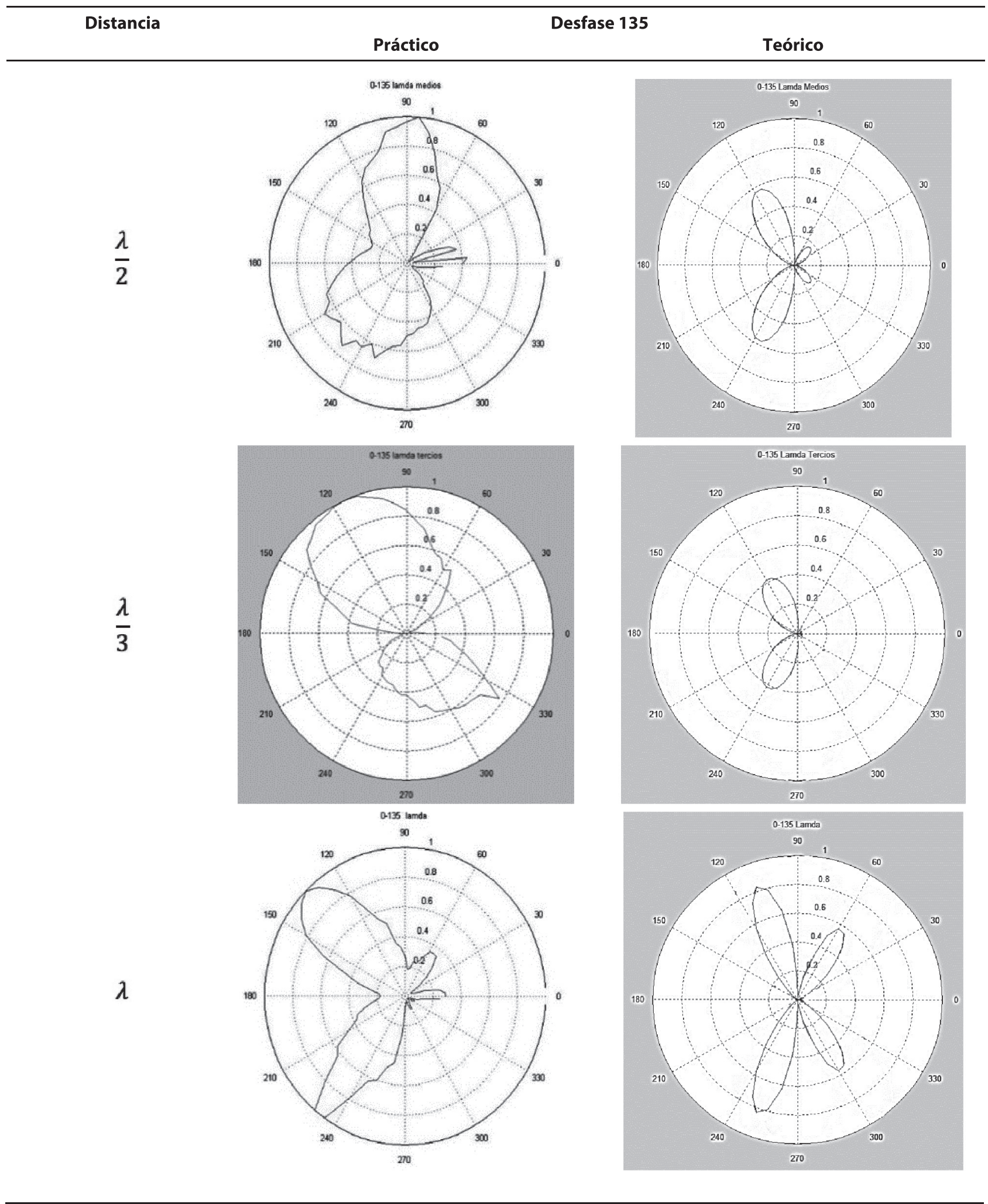

Fuente: Los autores 


\section{Conclusiones}

Durante el desarrollo del proyecto se pudo concluir que el arreglo realizado tuvo éxito, ya que, según las tablas de resultados, los patrones obtenidos son muy aproximados a los teóricos y se cumple el objetivo principal, el cual consistía en demostrar que se podía modelar y verificar el patrón de radiación variando características como distancia y fase. El arreglo puede ser empleado por el público en general y ser construido con materiales de fácil acceso.

Se observa que, para obtener unos resultados más ajustados a los teóricos, se debe tener en cuenta la exactitud del desfase entre las antenas; como este se realizó mediante un método gráfico, su exactitud no fue la ideal y esto afectó el patrón de radiación resultante. Uno de los elementos que influyó directamente en los resultados fue el generador de señales que trabaja a una intensidad de señal de solo $7 \mathrm{dBm}$ y al tener una pérdida por acoplamiento en el receptor de más de $9 \mathrm{dBm}$, sin contar con las pérdidas de espacio libre, el resultado varió con respecto al teórico.

Otro factor que afecta el desfase de los radiadores en un arreglo de antenas es la distancia entre el emisor y los receptores, ya que el arreglo se debe encontrar en el campo lejano de radiación de la antena transmisora para asegurar que el campo recibido sea lo más aproximado posible a una onda plana y el arreglo reciba la radiación de la onda prácticamente al mismo tiempo, esto con el objetivo de que el desfase en el arreglo sea lo más aproximado al teórico posible. Al tener una radiación a una potencia tan pequeña, la distancia a la cual se pudieron ubicar los receptores no fue la adecuada, ya que en el campo lejano la recepción era muy baja y casi nula; por tal motivo fue necesario ubicar el arreglo más cerca de la fuente, por lo cual los desfases no fueron los teóricos, ya que la onda de recepción no era una onda totalmente plana. Entre mayor es la frecuencia es más difícil realizar el desfase mediante el método de carta de Smith, ya que la longitud de onda es más pequeña y el corte que se debe realizar en el cable es también más reducido y compromete su exactitud; por ello es recomendable utilizar las diferentes matrices y componentes como la matriz de Butler.

\section{Referencias}

Aleixandre, X., Muñoz, J., Atienza, M. \& Navarro, A. (2012). Líneas de transmisión, Fisica II.( pp. 66-80) Catalunya: Universidad Oberta de Catalunya. Recuperado de https://www. exabyteinformatica.com/uoc/Fisica/Fisica_II_ES/Fisica_II_ ES_\%28Modulo_6\%29.pdf
Amanogawa.com. (2016). SEMCHIP. Recuperado de http:// www.amanogawa.com/

Anritsu. (2008). VNA Master. Recuperado de http://dl.cdnanritsu.com/en-us/test-measurement/files/BrochuresDatasheets-Catalogs/Brochure/11410-00400.pdf

Anritsu. (2009). Spectrum Master. Recuperado de https://dl.cdnanritsu.com/en-us/test-measurement/files/BrochuresDatasheets-Catalogs/Brochure/11410-00412.pdf

Danilo, A. (2016). Diseño e implementación de una matriz de Butler 4x4. Cuenca, Ecuador: Universidad de Cuenca.

Instituto Politecnico Nacional. (2016). Sistemas radiantes. Recuperado de http://antenaruval.mex.tl/ imagesnew2/0/0/0/2/0/5/4/9/3/1/TEMA\%203_1.pdf

Semchip. (s.f.). Amanogawa. Recuperado de http://www. amanogawa.com/index.html

Solid Signal, Signal Group LLC \& Affiliates. (2002). Solid Signal, Signal Group LLC \& Affiliates. Recuperado de http://www. solidsignal.com/pview.asp?p=cp2534 